\title{
Are multiple visual short-term memory storages necessary to explain the retro-cue effect?
}

\author{
Tal Makovski
}

Published online: 14 March 2012

(C) Psychonomic Society, Inc. 2012

\begin{abstract}
Recent research has shown that change detection performance is enhanced when, during the retention interval, attention is cued to the location of the upcoming test item. This retro-cue advantage has led some researchers to suggest that visual short-term memory (VSTM) is divided into a durable, limited-capacity storage and a more fragile, high-capacity storage. Consequently, performance is poor on the no-cue trials because fragile VSTM is overwritten by the test display and only durable VSTM is accessible under these conditions. In contrast, performance is improved in the retro-cue condition because attention keeps fragile VSTM accessible. The aim of the present study was to test the assumptions underlying this two-storage account. Participants were asked to encode an array of colors for a change detection task involving no-cue and retro-cue trials. A retro-cue advantage was found even when the cue was presented after a visual (Experiment 1) or a central (Experiment 2) interference. Furthermore, the magnitude of the interference was comparable between the no-cue and retro-cue trials. These data undermine the main empirical support for the two-storage account and suggest that the presence of a retro-cue benefit cannot be used to differentiate between different VSTM storages.
\end{abstract}

Keywords Visual short-term memory . Change detection . Retro-cue · Fragile VSTM

\footnotetext{
T. Makovski

Department of Psychology and Center for Cognitive Sciences, University of Minnesota,

Twin Cities, MN, USA

T. Makovski $(\triangle)$

Department of Psychology,

The College of Management Academic Studies,

7 Yitzhak Rabin Boulevard,

Rishon LeZion 75910, Israel

e-mail: tal.makovski@gmail.com
}

Almost every action we take in daily life depends on the use of visual memory. Visual memory retains information after it is no longer in view for durations ranging from a few milliseconds to a lifespan. It is often subdivided on the basis of duration and durability. Iconic memory holds a relatively large amount of information briefly (<500 ms; Sperling, 1960). Visual shortterm memory (VSTM) retains information in memory for several seconds, and unlike iconic memory, VSTM is typically considered a robust form of memory, but with limited storage capacity (Phillips, 1974).

Researchers have proposed that VSTM integrates highlevel cognitive processes with sensory processing (Johnson, Spencer, Luck, \& Schoner, 2009) and that its function is to retain visuospatial information to bridge across brief interruptions during ongoing processing (Conway, et al., 2005). Accordingly, there is evidence that some amount of visual information is retained after intervening visual or cognitive interference (Conway et al., 2005; Makovski, Shim, \& Jiang, 2006). Neurophysiological studies have further shown that neurons in the prefrontal lobe maintain their preference for specific visual properties during memory retention intervals (Miller, Erickson, \& Desimone, 1996), supporting the idea that unlike iconic memory, VSTM survives interference.

However, recent studies also have made it clear that visual or cognitive interference significantly disrupts VSTM. In typical VSTM tasks, people view an array of visual objects, and after a blank retention interval, they determine whether a test object is the same as one of the memory objects. When dynamic visual noise was presented during the retention interval, VSTM for colors was interrupted (Dent, 2010). That VSTM is disturbed by new visual input is further supported by the retrocue effect. Performance improves if, during the retention interval, a cue informs people which one of the memory objects will be tested (e.g., Griffin \& Nobre, 2003; Landman, Spekreijse, \& Lamme, 2003; Makovski \& Jiang, 2007; Makovski, Sussman, \& Jiang, 2008; Matsukura, Luck, \& Vecera, 2007; Sligte, 
Scholte, \& Lamme, 2008, 2009). Because the cue is presented after encoding, its impact is retrospective. Nonetheless, performance is better with retro-cue than with no cue. Researchers suggest that retro-cuing is effective because the cue protects VSTM from interference produced by the test display (Landman et al., 2003; Makovski et al., 2008) or from passive decay and interference by other uncued memory items (Matsukura et al. 2007). However, the underlying mechanism of the retro-cue advantage is not fully understood.

The vulnerability of VSTM to interference, as revealed by the retro-cue advantage, has led to the proposal that VSTM is not a unitary storage. Instead, an additional, intermediate VSTM storage may exist (Sligte et al., 2008, 2009; Sligte, Wokke, Tesselaar, Scholte, \& Lamme, 2011; Smith, Mollon, Bhardwaj, \& Smithson, 2011; Vandenbroucke, Sligte, \& Lamme, 2011). This proposal was supported by the finding that the estimates of memory capacity for line orientations nearly doubled in the retro-cue condition than in conditions where the cue was presented simultaneously with the test display (Landman et al., 2003). To account for this dramatic difference, it was suggested that performance in the no-cue condition is poor because it reflects the low capacity of a durable VSTM storage that is resistant to interference produced by new visual information (i.e., the test display). In contrast, with the use of a retro-cue, attention is summoned to keep accessible information held in a high-capacity, yet fragile VSTM storage (Sligte et al., 2008, 2009). Thus, according to this two-storage account, VSTM is composed of two separate memory storages that differ by several important characteristics. Fragile VSTM holds a large amount of visual information in a long-lasting, high-capacity but fragile memory buffer. This storage resembles iconic memory in its vulnerability to interference but does not depend on afterimages and is more limited in capacity than is iconic memory. When new visual information is presented, fragile VSTM is overwritten, and a limited amount of information is remained in a durable VSTM storage (Sligte et al., 2008, 2009).

The goal of the present study was to study the retro-cue advantage in order to better understand the subdivision of VSTM. The existence of a high-capacity VSTM stage was recently criticized by Matsukura and Hollingworth (2011), who found little evidence that VSTM exceeds typical capacity estimates. This study examined another key assumption underlying the two-storage account, which postulates that the retrocue manipulation differentiates between fragile and durable VSTM storages. This was accomplished by asking whether a retro-cue advantage is eliminated when the cue is introduced after visual or cognitive interference, at a time when fragile VSTM is supposedly erased. Participants encoded an array of colors in VSTM. ${ }^{1}$ Then an irrelevant color array was shown to

\footnotetext{
${ }^{1}$ Memory for colors, rather than line orientations, was tested to minimize grouping/chunking effects (Matsukura et al., 2007).
}

produce visual interference, or a cognitive task was carried out to produce central interference. According to the two-storage account, after interference only durable VSTM is available, and there is little to no fragile memory left. If the retro-cue is purported to salvage fragile memory (Sligte et al., 2008), its impact after interference (after fragile memory is disrupted) should be abolished. This prediction was tested in two experiments that introduced either visual (Experiment 1) or central (Experiment 2) interference prior to retro-cuing.

\section{Experiment 1}

Makovski and Jiang (2007) found that an irrelevant visual display impaired performance when attention was distributed across multiple memory items. However, the same display did not interfere with performance when presented after attention had been focused on a single memory item. These results supported the view that a retro-cue protects VSTM from interference. Notably, these data do not address the question of whether a retro-cue is still effective after interference, because the lack of an interference-with-no-cue control. Consequently, the finding that a retro-cue presented after interference was not better than a no-cue condition (Makovski \& Jiang, 2007, Experiment 1a) cannot be interpreted as saying that there is no retro-cue advantage after interference, because the two conditions differ by two critical factors (interference, cue). For instance, it is possible that the retro-cue advantage was overridden by the interference display. Similarly, Sligte et al. (2008) reported that an interference array appearing before the presentation of the retro-cue impaired performance in that condition. However, the lack of an interference-withno-cue baseline prevents one from drawing any conclusion regarding whether retro-cues are still usable after interference.

Participants were tested in a color change detection task, involving retro-cue and no-cue trials. No cues, rather than neutral cues, were used in order to minimize additional visual interference from the cues themselves. The critical question was whether a retro-cue advantage would be observed even when visual interference had occurred before the delivery of the cue. If only limited-capacity durable VSTM is available after interference (Sligte et al., 2008), no retro-cue advantage should be found after interference, and the retro-cue effect should be greater in the no-interference than in the interference condition. Alternatively, if attention protects whatever is currently being held in VSTM regardless of whether memory has been interfered with, the retro-cue advantage should be the same for the interference and no-interference conditions.

\section{Method}

Participants Students from the University of Minnesota participated in an IRB-approved study for extra course 
credits or payment. They were 18 to 25 years old and had normal or corrected-to-normal visual acuity. Eighteen participants (mean age, 19.9 years) completed Experiment 1.

Apparatus Participants were tested individually in a room with normal interior lighting. They sat unrestrained approximately $55 \mathrm{~cm}$ away from a 19 -in. CRT monitor. The experiments were programmed with Psychtoolbox (Brainard, 1997; Pelli, 1997) implemented in MATLAB (http://www.mathworks.com).

Stimuli and procedure Each trial began with a white fixation circle $\left(0.4^{\circ}\right.$ in diameter) presented against a black background for $500 \mathrm{~ms}$. The memory array was then presented for $500 \mathrm{~ms}$ and was composed of four unique colors randomly selected, without replacement, from nine distinct colors (orange, red, green, blue, white, yellow, purple, brown, and azure). The memory items were filled circles $\left(1.6^{\circ} \times 1.6^{\circ}\right)$, placed equidistantly $4.0^{\circ}$ from fixation.

The retention interval lasted for $2,000 \mathrm{~ms}$ and was followed either by a retro-cue or by the test display (no-cue trials; see Fig. 1a). The retro-cue was a white, central arrow $\left(1.6^{\circ}\right.$ in length) presented for $100 \mathrm{~ms}$ and was followed by a $400-\mathrm{ms}$ blank interval, after which the test display was presented (Fig. 1b). Participants were told that the item cued by the central arrow would always be the one tested. The interference trials were identical to the no-interference trials, except that four multicolored circles $\left(4.0^{\circ} \times 4.0^{\circ}\right)$ were presented for $500 \mathrm{~ms}$ during the retention interval. The interference array appeared evenly and randomly 500,650 , or $800 \mathrm{~ms}$ after the offset of the memory display, and its colors differed from the colors of the memory and test items (Fig. 1c, d).

Participants were told that the interference array, when presented, was irrelevant and that their task was to determine whether the test item was the same (press "s") or different (press " $d$ ") from the memory item previously displayed at the tested location. On half of the trials, the test item was the same as the memory item at that location. On the other half of the trials, it was a new color not presented previously on that trial. Only accuracy was emphasized, and a feedback in the form of a word ("correct" or "wrong") was presented after each response for $400 \mathrm{~ms}$. To reduce verbal recoding, participants were asked to repeat out loud a three-letter word as quickly as they could throughout a block of 15 trials.

Design To reduce uncertainty about the trial's sequence, the interference and no-interference trials were tested in separate blocks. ${ }^{2}$ Each participant performed four blocks of interference

\footnotetext{
${ }^{2}$ A pilot experiment using a mixed design found a similar pattern of data. However the retro-cue advantage was smaller under these conditions, probably because the conflicting requirements (ignore the interference array but attend to the cue) weakened the efficiency of the cue.
}

and no-interference trials in an $\mathrm{ABAB}$ order (counterbalanced across participants). Each block consisted of 60 trials, randomly and evenly divided into two cue conditions (retro-cue or no cue) and two response types (same or different). To familiarize participants with the task and displays, all participants completed 15 practice trials of the interference condition at the beginning of the experiment.

\section{Results}

Statistical results were generally the same for percent correct, $A^{\prime}$, and $K$ (Cowan, 2001). Percent correct data are reported below, while $A^{\prime}$ and $K$ data are listed in the Appendix.

Figure 2 depicts change detection performance as a function of interference and cue. A repeated measures ANOVA using these factors (interference vs. no interference; no cue vs. retrocue) revealed a retro-cue advantage: Retro-cue trials were more accurate than no-cue trials, $F(1,17)=18.06, p<.001, \eta_{\mathrm{p}}{ }^{2}=.52$. The effect of interference was also significant, since interference trials were less accurate than no-interference trials, $F(1$, 17) $=6.87, p<.02, \eta_{\mathrm{p}}{ }^{2}=.29$. No interaction was found between interference and cue, $F(1,17)=1.37, p>.25$. Reliable retro-cue advantages were found both when the interference array was presented, $t(17)=2.15, p<.05$, and when it was absent, $t(17)=3.39, p<.01$.

\section{Discussion}

Experiment 1 demonstrates that people can effectively use a retrospective cue to enhance VSTM performance, even when the cue was delivered after memory had been interfered with by new visual information. These data undermine the use of a retro-cue benefit as an index of two separate VSTM storages. According to Sligte et al. (2008), once new information is presented, fragile VSTM is erased. Given that the retro-cue is purported to salvage fragile VSTM, it should no longer be effective after interference, since there is nothing to salvage from. Instead, Experiment 1 showed that the retro-cue advantage persists even when the cue is delivered after the interference array. This suggests that the presence of a retro-cue advantage cannot be used as evidence for differentiating fragile VSTM from robust VSTM.

It is important to note that the retro-cue advantage observed in both interference and no-interference conditions cannot be attributed to differences in the comparison/retrieval process, because in both no-cue and retro-cue conditions, only a single comparison between memory and test had to be made. Additionally, the retro-cue advantage cannot be accounted for by differences in the retention interval because, if anything, the overall retention interval was longer in the retro-cue condition than in the no-cue condition (Makovski et al., 2008). 
Fig. 1 Schematic illustration of the conditions tested in Experiment 1

\section{a}

No-Cue

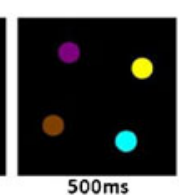

No-interference trials

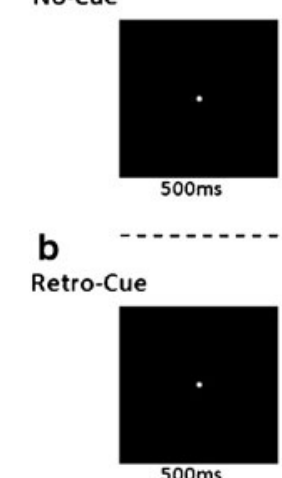

$500 \mathrm{~ms}$

C

No-Cue

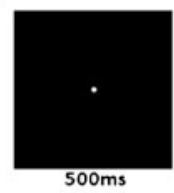

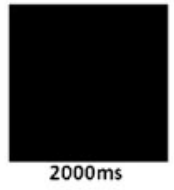

Interference trials

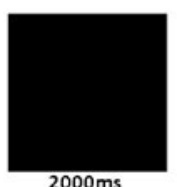

$2000 \mathrm{~ms}$

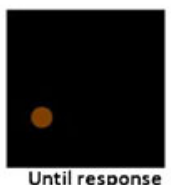

Until response

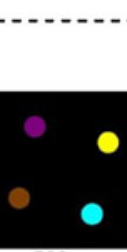

$500 \mathrm{~ms}$

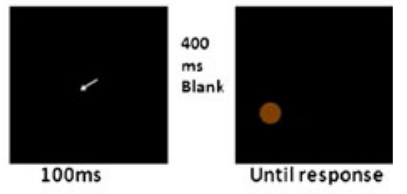

Until response

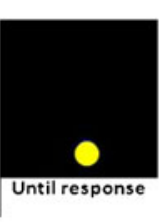

$2000 \mathrm{~ms}$
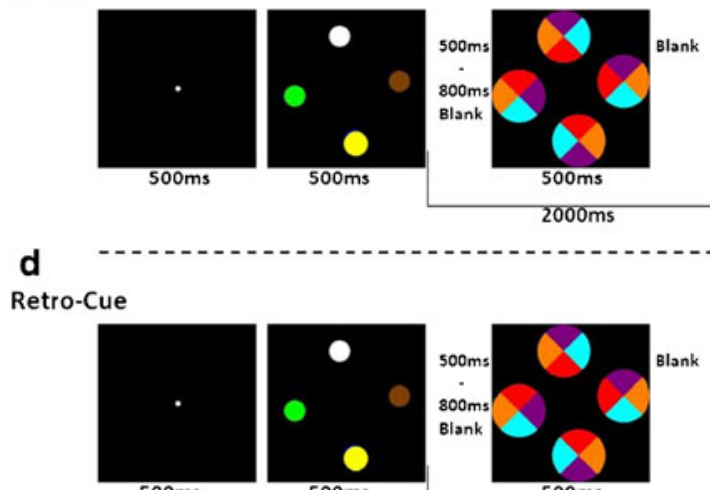

$500 \mathrm{~ms}$
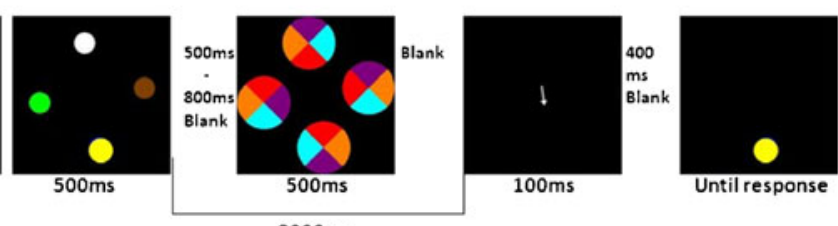

Can these results be generalized to other set sizes? This question is important because retro-cue effects may be more noticeable when memory load is higher (Sligte et al., 2008). Seventeen participants were tested in a follow-up experiment

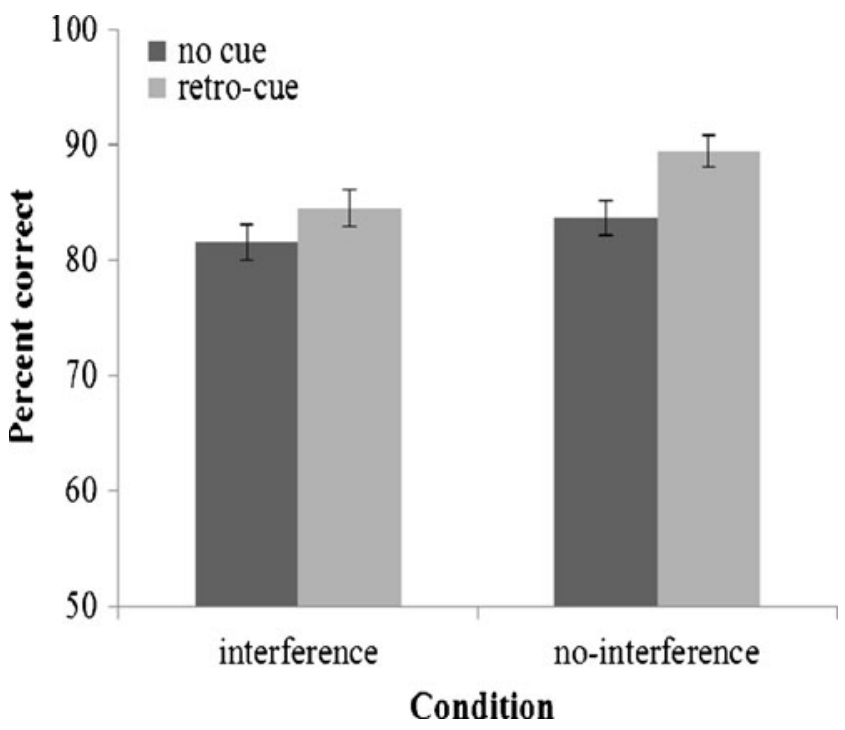

Fig. 2 Experiment 1's change detection results. Error bars show \pm 1 standard error of the mean that was identical to Experiment 1, except that the memory sets size was increased to six, well above capacity limit for colors (Alvarez \& Cavanagh, 2004). The results replicated Experiment 1's main findings. A significant retro-cue advantage was found in the interference condition $(72.3 \%$ vs. $69.0 \%, p<.02)$. The effect did not reach statistical significance in the no-interference condition $(73.3 \%$ vs. $70.3 \%$, $p=.16$ ), but there was no interaction between cue and interference, $F<1$. Thus, retro-cue benefits performance after interference even under high-load conditions.

\section{Experiment 2}

Experiment 1 showed that retro-cuing remained effective even after visual interference during VSTM retention interval. These data were not predicted on the basis of the twostorage account. Experiment 2 provides additional evidence using central, rather than visual, interference. This is necessary because the impact of visual interference on VSTM was modest in magnitude (see also Makovski et al., 2006). This may have left some fragile VSTM information intact. Experiment 2 was designed to test whether a retro-cue advantage would be found after stronger interference. This 
experiment took advantage of the finding that performing a secondary task during the retention interval of a change detection task is more destructive than just viewing an array of irrelevant visual stimuli (Makovski et al., 2006). Therefore, in the interference condition of Experiment 2, participants performed a speeded cognitive task during the retention interval; they classified a digit as odd or even. The no-interference trials were identical to before, and thus, interference in this experiment could be the product of new visual input, larger cognitive demand, and the need to make a motor response. Two questions were examined: whether retro-cuing would continue to be effective after intense interference, and whether the interference would be significantly larger in the retro-cue condition than in the no-cue condition.

\section{Method}

Participants Fifteen new participants (mean age, 19.5 years) completed Experiment 2.

Apparatus, stimuli, procedure, and design The experiment was identical to Experiment 1, except that an odd-even task was administered during the interference trials. Specifically, $450 \mathrm{~ms}$ after the removal of the memory array, a single white digit (font: "Times," 48) appeared at the center of the screen for $200 \mathrm{~ms}$. The digit was randomly selected from $0-9$, and participants were asked to respond as quickly and as accurately as possible whether it was odd (press "1") or even (press "2"). Participants had a total of $1,550 \mathrm{~ms}$ from the onset of the digit to respond (resulting in a total 2,000-ms retention interval). Feedback in the form of a red minus or a green plus was given for $400 \mathrm{~ms}$ immediately after the change detection response. Each participant was given 15 practice trials in both the oddeven task and the change detection task, before practicing on the combined tasks.

\section{Results and discussion}

One participant was excluded from the analysis due to chance performance in the odd-even task. Accuracy in the odd-even task for the remaining participants was $90.1 \%(S D=5.8)$, with an averaged response time of $663 \mathrm{~ms}(S D=96)$.

Figure 3 shows change detection performance as a function of interference and cue. A repeated measures ANOVA using these factors replicated the previous findings. First, retro-cue trials were more accurate than no-cue trials, $F(1,13)=12.32$, $p<.01, \eta_{\mathrm{p}}{ }^{2}=.49$, observed power $=.9$. Additionally, the new interference task was successful in taxing memory, since interference trials were substantially less accurate than nointerference trials, $F(1,13)=39.78, p<.001, \eta_{\mathrm{p}}{ }^{2}=.75$, observed power $=1.0$. Nevertheless, in spite of the strong interference, still no interaction was found between interference

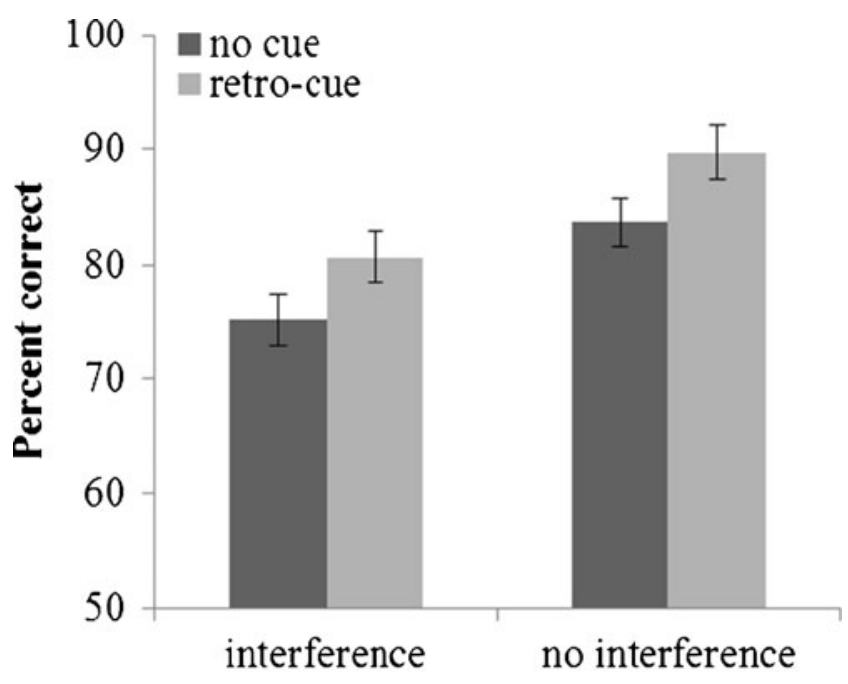

\section{Condition}

Fig. 3 Experiment 2's change detection results. Error bars show \pm 1 standard error of the mean

and cue, $F<1$. Participants were able to utilize the retro-cue in both the interference condition, $t(13)=2.34, p<.05$, and the no-interference condition, $t(13)=3.37, p<.01$. The interference effect was the same on both retro-cue trials (which allegedly taps into fragile VSTM) and no-cue trials (which allegedly taps into durable VSTM).

Across experiments, analysis confirmed that the interference effect was stronger in Experiment 2 than in Experiment 1, $F(1,30)=7.42, p<.01, \eta_{\mathrm{p}}{ }^{2}=.20$, with no other effects of experiment (all $p \mathrm{~s}>.23$ ). Thus, these results replicate and extend Experiment 1's finding that a retro-cue is as effective after interference as it is before interference.

\section{General discussion}

A two-storage account of the retro-cue advantage has suggested that two separate storage mechanisms coexist and that these storage mechanisms critically differ in their durability and capacity. The main empirical support for the two-storage account is the retro-cue advantage. It was proposed that the nocue condition taps into durable VSTM, whereas the retro-cue condition taps into fragile VSTM (Sligte et al., 2008, 2009). In contrast, the results of this study show that retro-cuing is effective even after visual or cognitive interference when supposedly only durable VSTM remains. Moreover, interference was not statistically different in the no-cue trials, as compared with the retro-cue trials. While these findings do not refute the possible existence of multiple VSTM storages, they challenge the notion that the retro-cue manipulation operationally differentiates between them (Sligte et al., 2008, 2009; Sligte et al., 2011; Vandenbroucke et al., 2011). To accommodate these data, the two-storage account would have to assume that 
retro-cue could be used to help both the fragile and the durable memory stores, to the same degree. This assumption, however, undermines the main empirical support that had initially motivated the division of VSTM into a fragile and a durable form.

That the retro-cue advantage was constant regardless of interference supports, instead, a more parsimonious explanation for the retro-cue advantage that does not postulate additional memory storage. Namely, people can take advantage of the cue to orient attention to an internal representation (Griffin \& Nobre, 2003), which consequently stabilizes this representation and protects it from interference (Makovski et al., 2008; Matsukura et al., 2007). Under this interpretation, fragility of VSTM representations is not all-or-none, and it is not necessary to propose two separate VSTM storages. It is only necessary to assume that the fragility of VSTM representations is graded and depends on attention. When attention is distributed across multiple items, memory is more fragile. When attention is focused on a subset of these items, memory becomes robust (Makovski \& Jiang, 2007). While this view resembles the two-storage account in that the retro-cue advantage reflects differences in VSTM durability, it differs from it in two key aspects. First, the nature of VSTM storage does not depend on whether it is probed with a retro-cue or a no-cue trial. Second, what attention does is to shield whatever VSTM currently holds, rather than to move items from one memory storage into another.

The present set of data raises another theoretically important question: What is the "proper" way to measure VSTM capacity? Here, color STM capacity, as indexed by $K$ (Cowan, 2001), ranged from 2.0 to 3.2, even though the same stimuli and retention interval were used in all conditions. That is, on the one hand, the "effective" capacity to perform the change detection task in the retro-cue, no-interference condition was estimated to be $\sim 3.2$ items. However, as others have noted, performance in the change detection task is not solely determined by VSTM capacity, and other mechanisms, such as sensory priming, long term memory, or central executive functions, can contribute to this task (Hollingworth, 2003; Makovski et al., 2006). On the other hand, these data might suggest that the number of colors that were actually available for performance in the face of distraction was only two. Indeed, this conservative estimate seems to better capture the notion of visual working memory as a top-down, online memory buffer that is readily available while engaging in other cognitive processing (Conway et al., 2005).

This wide range of capacity estimates is also in accord with other research arguing against a fixed and rigid VSTM capacity (e.g., Bays \& Husain, 2008). Instead, it suggests that representations held in VSTM are in constant flux and can be either stabilized by attention or interfered with by new visual input and increased cognitive load (Makovski, Watson, Koutstaal, \& Jiang, 2010). Whether such interference causes gradual degradation of resolution or completely terminates items from memory (Zhang \& Luck, 2009) is an important question for future research.

Acknowledgements This study was supported by funding from the University of Minnesota. I thank Lily Berrin for help with data collection and Yuhong V. Jiang and Khena M. Swallow for helpful discussions and comments.

\section{Appendix}

Table 1 Mean $A^{\prime}$ and Cowan's $K$ (with standard errors in parentheses) in Experiments 1 and 2: $K=$ memory load (i.e., 4$) \times($ hit + correct rejection -1$)$

\begin{tabular}{lllll}
\hline & \multicolumn{2}{l}{ Interference } & \multicolumn{2}{l}{ No Interference } \\
\hline Experiment 1 & No Cue & Retro-Cue & No Cue & Retro-Cue \\
$A^{\prime}$ & $.89(.01)$ & $.91(.01)$ & $.90(.01)$ & $.94(.008)$ \\
$K$ & $2.5(0.1)$ & $2.8(0.1)$ & $2.7(0.1)$ & $3.2(0.1)$ \\
Experiment 2 & & & & \\
$A^{\prime}$ & $.84(.02)$ & $.88(.02)$ & $.90(.02)$ & $.94(.02)$ \\
$K$ & $2.0(0.2)$ & $2.5(0.2)$ & $2.7(0.2)$ & $3.2(0.2)$ \\
\hline
\end{tabular}

\section{References}

Alvarez, G. A., \& Cavanagh, P. (2004). The capacity of visual shortterm memory is set both by visual information load and by number of objects. Psychological Science, 15, 106-111.

Bays, P. M., \& Husain, M. (2008). Dynamic shifts of limited working memory resources in human vision. Science, 321, 851-854.

Brainard, D. H. (1997). The psychophysics toolbox. Spatial Vision, 10 (4), 433-436.

Conway, A. R., Kane, M. J., Bunting, M. F., Hambrick, D. Z., Wilhelm, O., \& Engle, R. W. (2005). Working memory span tasks: A methodological review and user's guide. Psychonomic Bulletin \& Review, 12, 769-786.

Cowan, N. (2001). The magical number 4 in short-term memory: A reconsideration of mental storage capacity. The Behavioral and Brain Sciences, 24, 87-114.

Dent, K. (2010). Dynamic visual noise affects visual short-term memory for surface color, but not spatial location. Experimental Psychology, 57, 17-26.

Griffin, I. C., \& Nobre, A. C. (2003). Orienting attention to locations in internal representations. Journal of Cognitive Neuroscience, 15, $1176-1194$.

Hollingworth, A. (2003). Failures of retrieval and comparison constrain change detection in natural scenes. Journal of Experimental Psychology: Human Perception and Performance, 29, 388-403.

Johnson, J. S., Spencer, J. P., Luck, S. J., \& Schoner, G. (2009). A dynamic neural field model of visual working memory and change detection. Psychological Science, 20, 568-577.

Landman, R., Spekreijse, H., \& Lamme, V. A. (2003). Large capacity storage of integrated objects before change blindness. Vision Research, 43, 149-164.

Makovski, T., \& Jiang, Y. V. (2007). Distributing versus focusing attention in visual short-term memory. Psychonomic Bulletin \& Review, 14, 1072-1078. 
Makovski, T., Shim, W. M., \& Jiang, Y. V. (2006). Interference from filled delays on visual change detection. Journal of Vision, 6, 1459-1470.

Makovski, T., Sussman, R., \& Jiang, Y. V. (2008). Orienting attention in visual working memory reduces interference from memory probes. Journal of Experimental Psychology: Learning, Memory, and Cognition, 34, 369-380.

Makovski, T., Watson, L. M., Koutstaal, W., \& Jiang, Y. V. (2010). Method matters: Systematic effects of testing procedure on visual working memory sensitivity. Journal of Experimental Psychology: Learning, Memory, and Cognition, 36, 1466-1479.

Matsukura, M., \& Hollingworth, A. (2011). Does visual short-term memory have a high-capacity stage? Psychonomic Bulletin \& Review, 18, 1098-1104.

Matsukura, M., Luck, S. J., \& Vecera, S. P. (2007). Attention effects during visual short term memory maintenance: Protection or prioritization? Perception \& Psychophysics, 69, 1422-1434.

Miller, E. K., Erickson, C. A., \& Desimone, R. (1996). Neural mechanisms of visual working memory in prefrontal cortex of the macaque. Journal of Neuroscience, 16, 5154-5167.

Pelli, D. G. (1997). The VideoToolbox software for visual psychophysics: Transforming numbers into movies. Spatial Vision, 10, 437442.
Phillips, W. A. (1974). On the distinction between sensory storage and short-term visual memory. Perception \& Psychophysics, 16, $283-$ 290.

Sligte, I. G., Scholte, H. S., \& Lamme, V. A. F. (2008). Are there multiple visual short-term memory stores? PloS One, 3, e1699.

Sligte, I. G., Scholte, H. S., \& Lamme, V. A. F. (2009). V4 activity predicts the strength of visual short-term memory representations. Journal of Neuroscience, 29, 7432-7438.

Sligte, I. G., Wokke, M. E., Tesselaar, J. P., Scholte, H. S., \& Lamme, V. A. F. (2011). Magnetic stimulation of the dorsolateral prefrontal cortex dissociates fragile visual short-term memory from visual working memory. Neuropsychologia, 49, 1578-1588.

Smith, W. S., Mollon, J. D., Bhardwaj, R., \& Smithson, H. E. (2011). Is there brief temporal buffering of successive visual inputs? Quarterly Journal of Experimental Psychology, 64, 767-791.

Sperling, G. (1960). The information available in brief visual presentations. Psychological Monographs: General and Applied, 74, 1-30.

Vandenbroucke, A. R. E., Sligte, I. G., \& Lamme, V. A. F. (2011). Manipulations of attention dissociate fragile visual short-term memory from visual working memory. Neuropsychologia, 49, $1559-1568$.

Zhang, W., \& Luck, S. J. (2009). Sudden death and gradual decay in visual working memory. Psychological Science, 20, 423-428. 